\title{
Effect of vehicle motion stability after impact/crash on traffic safety
}

\author{
Eligiusz Mieloszyk ${ }^{1, *}$, Anita Milewska ${ }^{1}$, and Stawomir Grulkowski ${ }^{1}$ \\ ${ }^{1}$ Gdańsk University of Technology, Faculty of Civil and Environmental Engineering, 11/12 \\ Narutowicza Str., 80-233 Gdańsk, Poland
}

\begin{abstract}
The article presents the application of general stability theory to the study of road traffic stability immediately after an impact (crash, collision). It turns out that when modelling a collision, vehicles can be treated as colliding masses and dynamical systems can be assigned to this phenomenon.
\end{abstract}

\section{Introduction}

Stability theory deals with the qualitative analysis of dynamic systems and can be used to study the motion stability. In particular, the effect of motion stability directly after impact (crash, collision) on traffic safety can be investigated - especially on the adjacent lane or adjacent lanes. The point is that after hitting a vehicle or other obstacle, the vehicles involved in this event remain on the same lane, i.e. they do not block the adjacent lane, which reduces the likelihood of another accident (Figure 1).

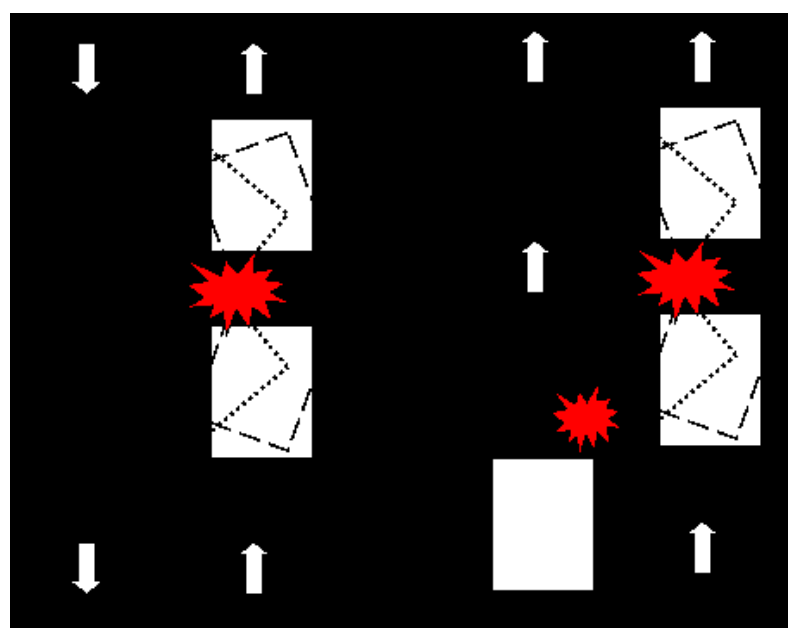

Fig. 1. Vehicle collision - "stable" and "unstable" (Vehicle direction may be same or opposite).

\footnotetext{
* Corresponding author: eligiusz.mieloszyk@pg.edu.pl
} 
This situation can be colloquially called "stable" in contrast to the "unstable" situation when the vehicles are on the adjacent lane or lanes. Thus, striving to avoid chaos, not only in the colloquial sense, but also in the sense of chaotic dynamics created by Henri Poincaré. Dynamical systems can have many attractors and it depends on the initial condition of a given orbit towards which attractor the system will converge [1]. Is it in the direction of chaotic motion, especially attracting chaotic motion? As in the case of solving numerically the algebraic equation $z^{3}-1=0$ using the Newton method. There are initial conditions in which all the solutions simultaneously "attract" and progression of approximations is not convergent with any of these solutions.

To shorten the description, it was assumed that there are motion assumptions and classes of functions describing the phenomenon, etc., allowing for subsequent mathematical notations and descriptions. These assumptions allow also to perform appropriate, necessary mathematical and mechanical operations.

The methods for assessment of the stability of dynamic systems include both indirect and direct approaches. The first group uses different forms of the system response, and the second group enables studying the stability with no prior information on the system response, which is its main advantage.

For the following vector differential equation

$$
\frac{d}{d t} \vec{x}=F(t, \vec{x})
$$

with the condition

$$
\vec{x}\left(t_{0}\right)=\overrightarrow{x_{0}}
$$

where $t \geq 0$, then the stable solutions are obtained.

There are many definitions of stability. However, in the case of the analysed problem, the following definition is used:

The solution of the equation (1) with the condition (2) is stable in the range $<t_{0}, \infty$ ), if for the arbitrary positive number $\varepsilon$, there is such a positive number $\delta$, that for each solution $\vec{y}$ of the equation (1) with the condition $\vec{y}\left(t_{0}\right)=\overrightarrow{y_{0}}$, that inequality $\left|\overrightarrow{y_{0}}-\overrightarrow{x_{0}}\right|<\delta$ is satisfied, the condition $|\vec{x}-\vec{y}|<\varepsilon$ is satisfied. The stability defined in such a way is called the Lapunov stability [2].

It must be noted here that many differential equations of the nth order that describe the motion, including vehicles motion, can be written using a vector differential equation of the first order in the form (1).

The problem (1), (2) can be used to describe the motion trajectory. In the case of $n=2$, the stability of trajectory indicates that in the phase space each trajectory starting in $t_{0}$ from the circle of the radius $\delta$ will not leave the circle of the radius $\varepsilon$ in any arbitrary time $t>t_{0}$. If this is not satisfied, the system is not stable, which is not advantageous from the practical point of view.

The process of vehicles colliding is an interaction between them, in which the impact time is very brief leading to a rapid change in their velocity and resulting in the creation of very large impact forces acting on vehicles [3]. It is a motion with impacts. The entire process can be divided into three phases: pre-impact motion, proper impact and after-impact.

Solution of the so-called collision issues, i.e. the problem of determining the trajectory and velocity after impact, and in particular determining its stability depending on the trajectory and velocity before impact are of great importance for determining the safety after a collision. 
In crash modelling the vehicles can be treated as colliding masses. In this case, the following can be written as in equation

$$
\begin{gathered}
m_{1} v_{1}^{1}+m_{2} v_{2}^{1}=m_{1} v_{1}+m_{2} v_{2} \\
v_{1}^{1}-v_{2}^{1}=R\left(v_{2}-v_{1}\right)
\end{gathered}
$$

where $v_{1}, v_{2}, v_{1}^{1}, v_{2}^{1}$ indicate the velocities of the vehicles after and before the crash.

$\mathrm{R}$ is called the coefficient of restitution, which is determined empirically and lies in the range $\langle 0,1\rangle$. It is the value that reflects he character of the impact and from the perspective of vehicle crash, it can be assumed that it is a number in the range $(0,1)$ because the collision is accompanied by losses of the kinetic energy of the vehicles.

\section{Generalised dynamic system in the process of vehicle collision}

The mechanical system shown in Fig. 2 can be used to model the collision between two vehicles, to which masses $m_{1}, m_{2}$ are assigned, respectively. The model also accounts for the local susceptibility of vehicles by including springs with elasticity coefficients $k_{1} i k_{2}$

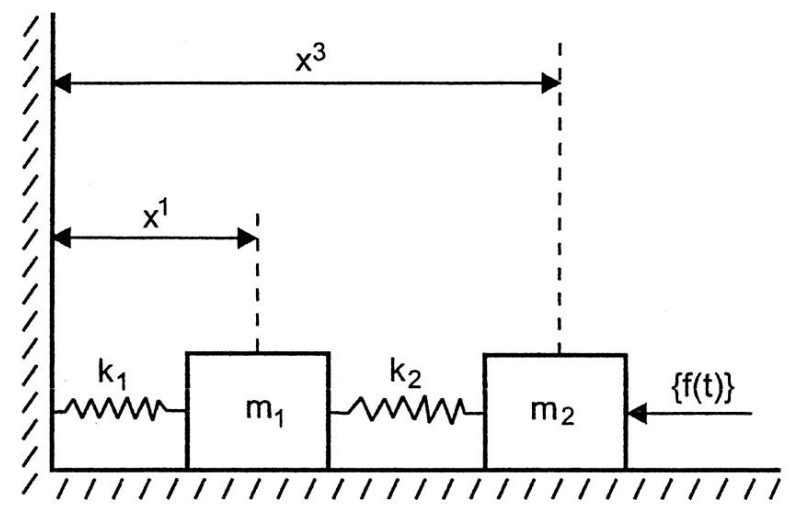

Fig. 2. Mechanical system [4].

Let's assume that the vehicle with the mass $m_{2}$ is subject to an external force $\{f(t)\}$ coming e.g. from the next vehicle, and the frictional resistance of the vehicles is proportional to the velocity, where $r_{1}, r_{2}$ are the friction coefficients of the vehicle with mass $m_{1}$ and $m_{2}$, respectively.

The resistance $r_{1}, r_{2}$ should be considered in connection with the tyre contact with the road surface on which they are rolling or on which braking takes place. These parameters are difficult to measure [5]. These parameters are determined both by the design and the materials the tyres are made of, as well as the properties of road surfaces, especially in terms of texture, evenness and adhesion. This causes serious complications when determining their value in a specific case, as well as in general, since road traffic involves very different types of tyre that roll on various surfaces. In addition, the tyre pressure has a significant effect on the length of the braking distance, and thus on the impact force. The shortest braking distances are obtained for normal tyre pressure. Incorrect tyre pressure to the vehicle manufacturer's recommendations can extend the braking distance by up to $20 \%$ [5].

The mechanical system from Fig. 2 can be described as in [4] with the following system of differential equations

$$
m_{1} \dot{x}^{2}+r_{1} x^{2}+k_{1} x^{1}+k_{2}\left(x^{1}-x^{3}\right)=0
$$




$$
m_{2} \dot{x}^{4}+r_{2} x^{4}+k_{2}\left(x^{3}-x^{1}\right)=\{f(t)\} ，
$$

in which $x^{2}=\dot{x}^{1}$ and $x^{4}=\dot{x}^{3}$.

It is a special case of the generalised dynamic system introduced in [4] with a description:

$$
S \vec{x}=\boldsymbol{A} \vec{x}+\boldsymbol{B} \vec{u}
$$

in which $S$ is a certain linear operation, while $\boldsymbol{A}$ and $\boldsymbol{B}$ are matrices of appropriate dimensions matching the response $\vec{x}=\left[x^{1}, x^{2}, \ldots, x^{n}\right]^{T}$ of the system and forced motion $\vec{u}$.

Assuming: $\left[x^{1}, x^{2}, x^{3}, x^{4}\right]^{T}$ ( $T$ is a transposition operation), $S=\frac{d}{d t}$, the last set of differential equations can be written in the form (3). As matrices $\boldsymbol{A}$ and $\boldsymbol{B}$, it is assumed:

$$
\boldsymbol{A}=\left[\begin{array}{cccc}
0 & 1 & 0 & 0 \\
-\frac{k_{1}+k_{2}}{m_{1}} & -\frac{r_{1}}{m_{1}} & \frac{k_{2}}{m_{1}} & 0 \\
0 & 0 & 0 & 1 \\
\frac{k_{2}}{m_{2}} & 0 & -\frac{k_{2}}{m_{2}} & -\frac{r_{2}}{m_{2}}
\end{array}\right] \quad, \quad \boldsymbol{B}=\left[\begin{array}{c}
0 \\
0 \\
0 \\
\frac{1}{m_{2}}
\end{array}\right]
$$

Forced motion $\vec{u}$ is $\{f(t)\}$.

From this, it follows that the stability of this system can be studied with the use of the Heaviside operator $p_{q^{-}}[4,6]$.

In [4], the formula for the only response of a generalised dynamic system (3) was proved with initial conditions. From this formula, it follows that the mechanical system response from Fig. 2 with initial conditions

$$
\vec{x}\left(t_{0}\right)=\left[\begin{array}{l}
x_{0}^{1}\left(t_{0}\right) \\
x_{0}^{2}\left(t_{0}\right) \\
x_{0}^{3}\left(t_{0}\right) \\
x_{0}^{4}\left(t_{0}\right)
\end{array}\right]
$$

is defined by a formula

$$
\vec{x}=\left[\begin{array}{c}
\frac{\Delta_{1}\left(p_{q}\right)}{\Delta\left(p_{q}\right)} \\
\frac{\Delta_{2}\left(p_{q}\right)}{\Delta\left(p_{q}\right)} \\
\frac{\Delta_{3}\left(p_{q}\right)}{\Delta\left(p_{q}\right)} \\
\frac{\Delta_{4}\left(p_{q}\right)}{\Delta\left(p_{q}\right)}
\end{array}\right], \text { where } \Delta\left(p_{q}\right)=\operatorname{det}\left(\boldsymbol{A}-p_{q} \boldsymbol{I}\right)
$$

whereas $\Delta_{i}\left(p_{q}\right), i=1,2,3,4$ are determinants of special matrices. Their detailed form is not important from the point of view of these considerations. It is enough to remember, as previously noted, that these are quantities dependent on the operator $p_{q}$. From our point of view, an important determinant is $\Delta\left(p_{q}\right)=\operatorname{det}\left(\boldsymbol{A}-p_{q} \boldsymbol{I}\right)$, because it determines the stability of the analysed system.

The formula (6) follows directly from the general formula for the response of the generalised dynamic system, in which for $n=4$ the formulas (4) and initial conditions (5) should be used. 
As results from the formula (6) and the formula (4) the $\operatorname{determinant} \operatorname{det}\left(\boldsymbol{A}-p_{q} \boldsymbol{I}\right)$ depends on $m_{i}, k_{i}, r_{i}, i=1,2$ so the answer $\vec{x}$, and more precisely its components are rational functions of the Heaviside operator $p_{q}$ additionally dependent on $m_{i}, k_{i}, r_{i}, i=$ 1,2 . If so, then these quantities, $m_{i}, k_{i}, r_{i}, i=1,2$, determine the stability of the modelled collision of vehicles. They include: vehicle masses, their impact susceptibility, tyre parameters, tyre pressure, types and properties of road surface, etc. These parameters "hidden" in matrix A have an effect on the stability and safety of motion after the impact analysed here.

\section{Trajectories of motion near singular points}

When a point moves along any path, at the position where the currently considered point is located, we can erect three planes to the path: an osculating plane, a perpendicular plane and a rectifying plane. The edges of intersection of these planes are determined by straight lines (axes): tangent, normal main and binormal.

It can be shown that the motion of a point takes place temporarily in an osculating plane, and therefore in further considerations it is enough to consider only the path in a plane (plane path), on which we enter the coordinates $x^{1}(t), x^{2}(t)$.

The system of differential equations

$$
\begin{aligned}
& \dot{x}^{1}=f_{1}\left(x^{1}, x^{2}\right) \\
& \dot{x}^{2}=f_{2}\left(x^{1}, x^{2}\right)
\end{aligned}
$$

for a moment $t$ determines the point $\left(x^{1}(t), x^{2}(t)\right)$.

Characteristics of vehicles, surfaces, etc. are included in the functions $f_{1}, f_{2}$.

Note. The system (5) is a special case of a generalised non-linear dynamic system - [4]. In subsequent moments of time, the states of the system will form a curve, which is the trajectory of motion. Each trajectory has its origin at the point determined by the initial conditions

$$
\left(x^{1}\left(t_{0}\right), x^{2}\left(t_{0}\right)\right)=\left(x_{0}^{1}, x_{0}^{2}\right) .
$$

Many different behaviours of the system due to its stability occur near singular points.

The singular point of the system (5) is the point which is the solution of the system of equations

$$
\begin{aligned}
& f_{1}\left(x^{1}, x^{2}\right)=0 \\
& f_{2}\left(x^{1}, x^{2}\right)=0 .
\end{aligned}
$$

It follows that the singular point $\left(x_{o s}^{1}, x_{o s}^{2}\right)$ satisfies the system of equations (6).

As in [4], the system can be linearised (5) and expressed in the form (3) using singular points. From this it follows that near a singular point a non-linear system of differential equations can be replaced by a linear system in which matrix $\mathrm{A}$ is of the form

$$
\boldsymbol{A}=\left[\begin{array}{ll}
\frac{\partial f_{1}}{\partial x^{1}} & \frac{\partial f_{1}}{\partial x^{2}} \\
\frac{\partial f_{2}}{\partial x^{1}} & \frac{\partial f_{2}}{\partial x^{2}}
\end{array}\right]
$$


whose elements are calculated, of course, in a singular point.

As it was shown in point 2 of this paper, the equation determines the stability of the system

$$
\operatorname{det}\left(\boldsymbol{A}-p_{q} \boldsymbol{I}\right)=\operatorname{det}\left[\begin{array}{cc}
\frac{\partial f_{1}}{\partial x^{1}}-p_{q} & \frac{\partial f_{1}}{\partial x^{2}} \\
\frac{\partial f_{2}}{\partial x^{1}} & \frac{\partial f_{2}}{\partial x^{2}}-p_{q}
\end{array}\right]
$$

or more precisely, its solution.

It turns out that near the singular point trajectories of motion can follow in a few strictly defined ways. It depends on the values of the expressions

$$
p=-\operatorname{tr} \boldsymbol{A}, q=\operatorname{det} \boldsymbol{A}
$$

defined for matrix A described by formula (7). They determine the stability of the collision and have a significant impact on traffic safety, especially on the adjacent lane or lanes.

The following cases can be distinguished:

I. A singular point is a node when $q>0$ and $p^{2}-4 p q<0$. In this case, the trajectories concentrically converge to the singular point and the node is stable or move away from it and the node is unstable (Fig.3). It is stable when additionally $p>0$ and unstable when $p<0$.

a)

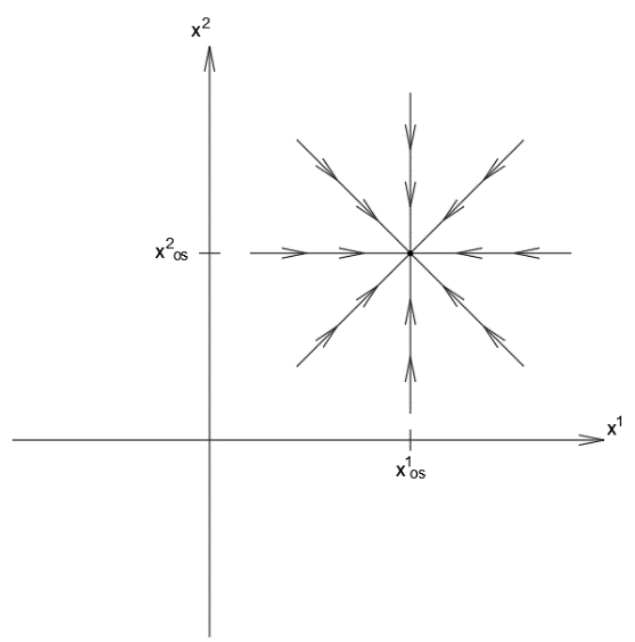

b)

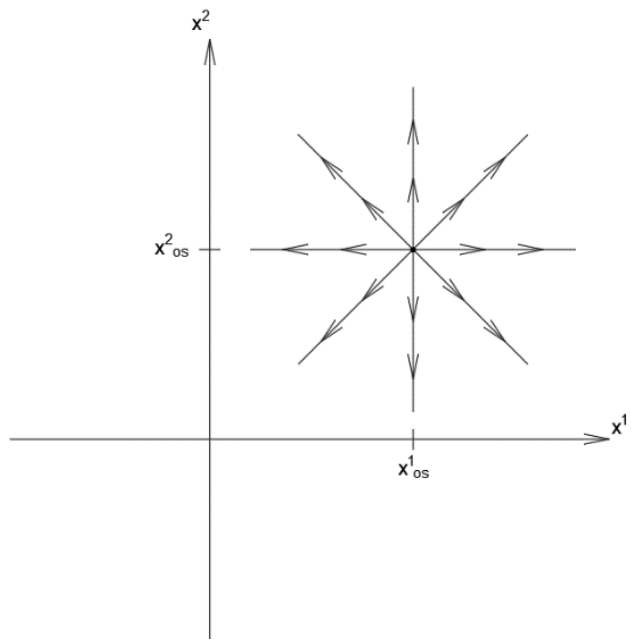

Fig. 3. Trajectories (behaviour of the vehicle) near the node: a) stable b) unstable.

II. The singular point is the focus when $q>0$ and $p^{2}-4 p q>0$. In this case, the trajectories concentrically converge to the singular point and the node is stable or diverge from it and the node is unstable - Fig.4. It is stable when additionally $p>0$ and unstable when $p<0$.
a)
b) 

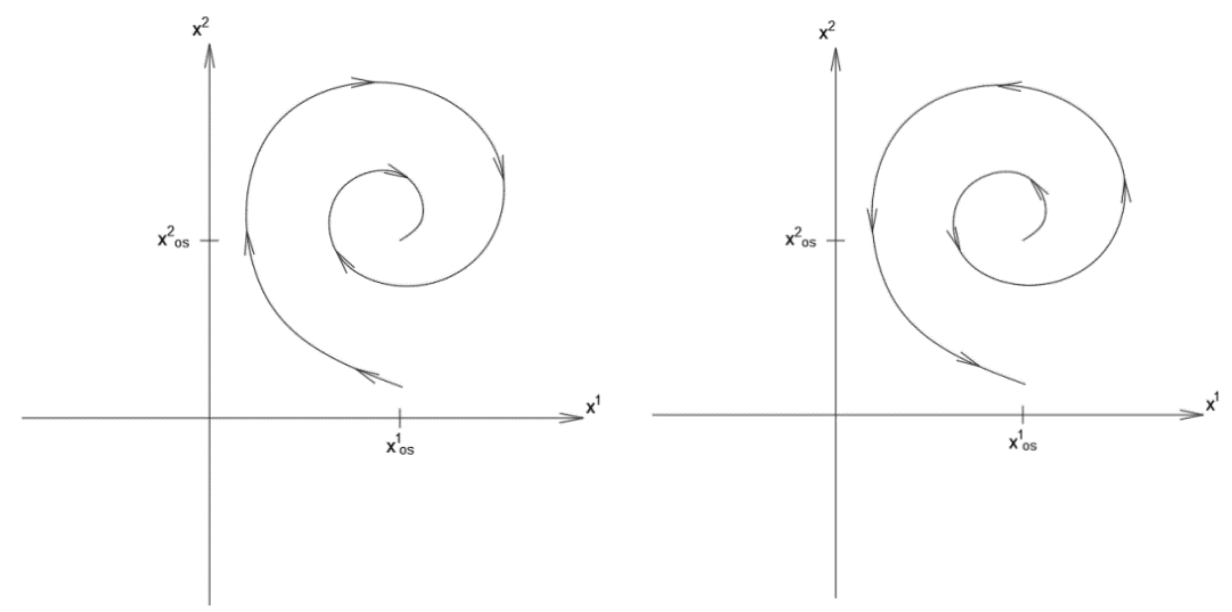

Fig. 4. Trajectories near the focus: a) stable b) unstable.

III. The singular point is a saddle when $q<0$. In this case, only two trajectories approach to the singular point. The others move away from it, or after a short approach, they begin to move away (Fig.5). Therefore, this point is not stable.

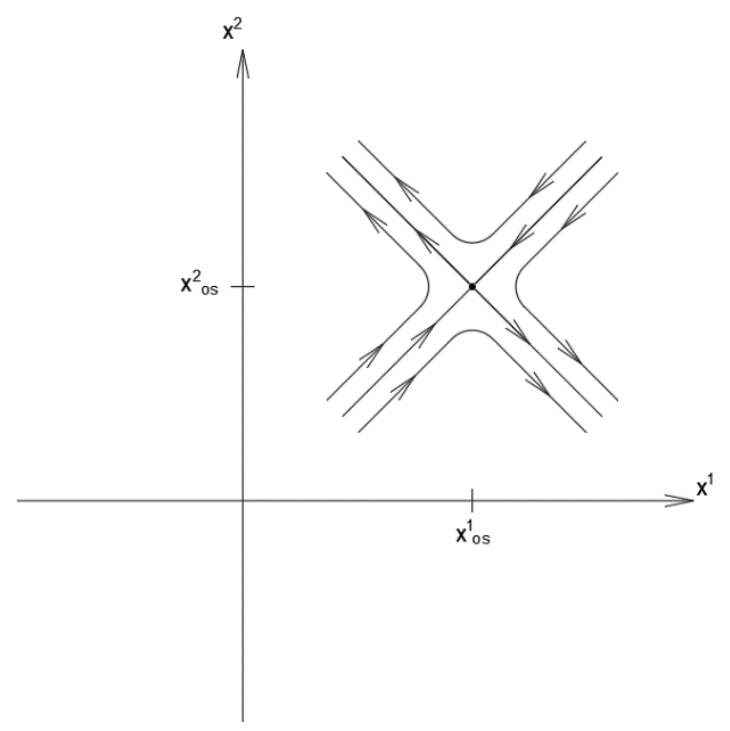

Fig. 5. Trajectories near the saddle.

IV. The singular point is a saddle-node, when $q=0$. In this case trajectories show the properties of the saddle and the stable or unstable node (Fig. 6). It can be good or bad, because a stable or unstable path may be struck. 


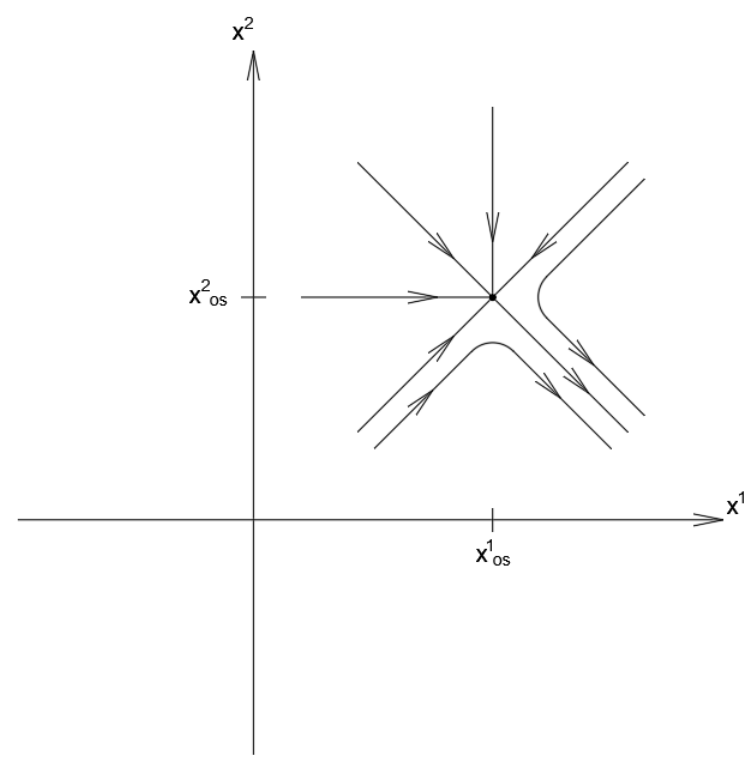

Fig. 6. Trajectories near the saddle and stable node.

Note. In the case I and II, this is also the asymptotic stability of Lapunov, i.e. with the passage of time $(\mathrm{t} \rightarrow \infty)$, the values of variables approximate the singular point.

The functions $f_{1}, f_{2}$ contain information about the system and the conditions of the collision. Parameters must be selected so that the system is stable: the condition of the tyres, their quality, materials they are made of, the condition of the surface, its texture, evenness, adhesion, crumple zone, etc. Then the chances of entering another lane during and after the collision definitely decrease.

\section{Conclusions}

The entire impact process (vehicle collision) can be divided into three phases: pre-impact motion, proper impact and after-impact.

It was indicated that the characteristics of vehicles, the tyres contact with the road surface on which the braking takes place, the materials of which the tyres are made, and the properties of road surfaces, especially in terms of texture, evenness and adhesion must be accounted for in the modelling process, as well as in the process of testing the stability of motion in various phases of crash (collision).

Stable cases are beneficial from the point of view of traffic safety.

Very different types of vehicle participate in road traffic, using the most diverse types of tyres with different technical conditions, which roll on various surfaces, so the safety of traffic is determined not only by stability but also by the stability margin.

\section{References}

1. E. Ott, Chaos in Dynamical Systems (Press Syndicate of the University of Cambridge, New York, 1994)

2. L.C. Evans, Partial differential equations (American Mathematical Society, 1998)

3. W. Szcześniak, M. Ataman, Selected problems of the classic theory of impact (in Polish), Logistyka. 6 pp. 3305-3310 (2010) 
4. E. Mieloszyk, Non-classical operational calculus in application to generalized dynamical systems (in Polish) (Polish Academy of Sciences Scientific Publishers, Gdansk, 2008)

5. R.S. Jurecki, T.L. Stańczyk, M.J. Jaśkiewicz, Driver's reaction time in a simulated, complex road incident, Transport. 32 pp. 44-54 (2014). doi:10.3846/16484142.2014.913535

6. E. Mieloszyk, Application of non-classical operational calculus to solving some boundary value problem, Integr. Transform. Spec. Funct. 9 pp. 287-292 (2000). doi:10.1080/10652460008819262

7. H.-N. Li, D.-S. Li, G.-B. Song, Recent applications of fiber optic sensors to health monitoring in civil engineering, Eng. Struct. 26 pp. 1647-1657 (2004). doi:10.1016/J.ENGSTRUCT.2004.05.018

8. B. Radziszewski, Elements of control theory (in Polish) (Publisher of the Silesian University of Technology, Kielce, 1999) 